\title{
Diagnostic Value of Plasma miR-181b, miR-196a, and miR-210 Combination in Pancreatic Cancer
}

\author{
Gongpan Liu, ${ }^{1}$ Cunhua Shao, ${ }^{1}$ Anyun Li, ${ }^{2}$ Xiaobin Zhang, ${ }^{1}$ Xingjun Guo, ${ }^{1}$ and Jiangong Li ${ }^{1}{ }^{1}$ \\ ${ }^{1}$ Department of Hepatobiliary Surgery, Dongying People's Hospital, No. 317, Nanyi Road, Dongying City, \\ Shandong Province 257091, China \\ ${ }^{2}$ Department of General Surgery, Dongying Honggang Hospital, No. 436, Huanghe Road, Dongying City, \\ Shandong Province 257000, China \\ Correspondence should be addressed to Jiangong Li; lijiangong248@163.com
}

Received 14 May 2020; Accepted 28 July 2020; Published 6 August 2020

Academic Editor: Kazuhiko Uchiyama

Copyright (c) 2020 Gongpan Liu et al. This is an open access article distributed under the Creative Commons Attribution License, which permits unrestricted use, distribution, and reproduction in any medium, provided the original work is properly cited.

Purpose. This study was aimed at investigating the roles of plasma miR-181b, miR-196a, and miR-210 in the diagnosis of pancreatic cancer (PC). Methods. Plasma samples were isolated from 40 patients with PC and 40 healthy individuals, respectively. The expression of miR-181b, miR-196a, and miR-210 was detected by qRT-PCR. The level of carbohydrate antigen 199 (CA199) was measured by an electrochemiluminescence (ECL) assay. The receiver operating characteristic (ROC) curve was used to analyze the diagnostic value of miR-181b, miR-196a, miR-210, CA199, and their combinations in PC. Results. The expression of plasma miR-181b, miR-196a, and miR-210 was significantly upregulated in PC patients. The plasma level of CA199 was also significantly increased in PC patients. The expression of miR-181b, miR-196a, and miR-210 was closely associated with lymph node metastasis, clinical stage, and vascular invasion but not correlated with age, gender, and tumor size. miR-181b, miR-196a, and miR-210 have lower AUC than CA199 in the diagnosis of PC. miR-181b+miR-210 and miR-196a+miR-210 also have lower AUC than CA199. It is worth noting that miR-181b+miR-196a+miR-210 has a higher AUC than CA199 in the diagnosis of PC. Conclusion. The combination of plasma miR-181b, miR-196a, and miR-210 had a good diagnostic value for PC.

\section{Introduction}

Pancreatic cancer (PC) is one of the most common gastrointestinal malignancies and the sixth leading cause of cancerrelated death in China $[1,2]$. Because early PC lacks specific clinical symptoms, most patients are already in advanced stages when they are diagnosed with PC [3]. Although the therapeutic techniques have greatly improved, the survival rate of PC patients remains poor [4]. The median survival time of $\mathrm{PC}$ is about 5 to 8 months, and the 5 -year survival rate is only about $8 \%$ [5]. Therefore, identifying specific biomarkers is very important for the diagnosis of PC, especially for PC at an early stage.

MicroRNAs (miRNAs) are a class of small endogenous noncoding RNAs with 18-25 nucleotides in length, which can modulate gene expression at posttranscriptional level $[6,7]$. More and more evidences have indicated that miRNAs exert important roles in the oncogenesis and metasta- sis of numerous tumors [8-10]; thus, the alteration of certain miRNAs may predict tumors to a certain extent. Under normal physiological condition, the levels of miRNAs are stable in the plasma. The abnormal expression of miRNAs has been detected in the plasma of patients with PC. Duell et al. have shown that plasma miR-10b, miR21-5p, miR-30c, and miR-106b are upregulated in PC patients, and those miRNAs may be biomarkers for PC screening [11]. miR-181b, miR-196a, and miR-210 are three important miRNAs that were involved in the tumorigenesis of PC. Zhou et al. have shown that the plasma level of miR$181 \mathrm{~b}$ is increased in patients with PC, which is correlated with tumor stage, lymph node metastases, and distant metastasis [12]. Xu et al. have found that exosome miR196a is elevated in the plasma of patients with PC, which acts as a potential indicator of localized PC [13]. Ho et al. have demonstrated that circulating miR-210 level is elevated in PC patients, which may serve as a biomarker for 


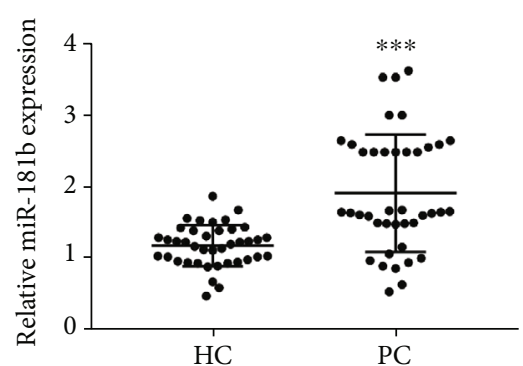

(a)

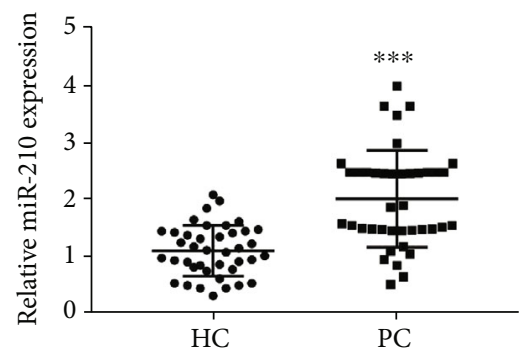

(c)

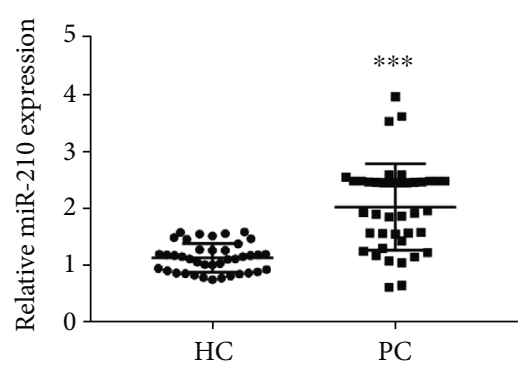

(b)

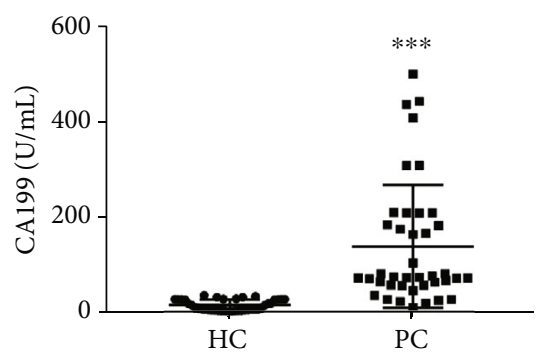

(d)

FIGURE 1: The levels of plasma miR-181b, miR-196a, miR-210, and CA199 in pancreatic cancer (PC) patients and healthy volunteers. (a) The expression of plasma miR-181b was detected by qRT-PCR. (b) The expression of plasma miR-196a was detected by qRT-PCR. (c) The expression of plasma miR-210 was detected by qRT-PCR. (d) The level of plasma CA199 was detected by the electrochemiluminescence assay. ${ }^{* * *} P<0.001$ vs. HC group.

TABLE 1: Correlation between the expression of plasma miR-181b, miR-196a, and miR-210 and clinical indicators in PC patients.

\begin{tabular}{|c|c|c|c|c|c|c|c|}
\hline Clinical indicators & Case & miR-181b expression & $P$ value & miR-196a expression & $P$ value & miR-210 expression & $P$ value \\
\hline Age (y) & 40 & & & & & & \\
\hline$<60$ & 21 & $1.940 \pm 0.833$ & 0.7603 & $2.040 \pm 0.808$ & 0.9349 & $2.020 \pm 0.851$ & 0.9901 \\
\hline$\geq 60$ & 19 & $1.860 \pm 0.810$ & & $2.020 \pm 0.722$ & & $2.02 \pm 0.861$ & \\
\hline \multicolumn{8}{|l|}{ Gender } \\
\hline Male & 22 & $1.830 \pm 0.773$ & 0.5477 & $1.940 \pm 0.692$ & 0.4376 & $1.930 \pm 0.796$ & 0.4633 \\
\hline Female & 18 & $1.990 \pm 0.895$ & & $2.130 \pm 0.840$ & & $2.130 \pm 0.911$ & \\
\hline \multicolumn{8}{|c|}{ Lymph node metastasis } \\
\hline Yes & 23 & $2.490 \pm 0.618$ & $0.0010^{* * *}$ & $2.290 \pm 0.618$ & $0.0008^{* *}$ & $2.050 \pm 0.511$ & $0.0013 * *$ \\
\hline No & 17 & $1.647 \pm 0.635$ & & $1.428 \pm 0.356$ & & $1.528 \pm 0.355$ & \\
\hline \multicolumn{8}{|l|}{ Clinical stage } \\
\hline I-II & 21 & $2.330 \pm 0.768$ & $0.0048^{* *}$ & $2.390 \pm 0.698$ & $0.0319^{*}$ & $2.520 \pm 0.730$ & $0.0027^{* *}$ \\
\hline III-IV & 19 & $1.620 \pm 0.630$ & & $1.820 \pm 0.611$ & & $1.670 \pm 0.623$ & \\
\hline \multicolumn{8}{|l|}{ Vascular invasion } \\
\hline Yes & 20 & $2.260 \pm 0.594$ & $0.0002^{* * *}$ & $2.340 \pm 0.479$ & $0.0016^{* *}$ & $2.230 \pm 0.533$ & $0.0019 * *$ \\
\hline No & 20 & $1.550 \pm 0.369$ & & $1.728 \pm 0.593$ & & $1.628 \pm 0.348$ & \\
\hline \multicolumn{8}{|l|}{ Tumor size $(\mathrm{cm})$} \\
\hline$<2$ & 14 & $1.930 \pm 0.884$ & 0.9703 & $2.040 \pm 0.838$ & 0.9779 & $2.050 \pm 0.973$ & 0.9722 \\
\hline $2-4$ & 13 & $1.930 \pm 0.818$ & & $2.050 \pm 0.723$ & & $2.030 \pm 0.801$ & \\
\hline$>4$ & 13 & $1.860 \pm 0.826$ & & $1.990 \pm 0.764$ & & $1.980 \pm 0.0 .804$ & \\
\hline
\end{tabular}

Notes: ${ }^{* *} P<0.01$ and ${ }^{* * *} P<0.001$.

diagnosis [14]. In addition, fecal miR-181b, miR-196a, and miR-210 are highly expressed in PC patients, and miR$181 \mathrm{~b}$ and miR-210 may be biomarkers for PC screening [15]. However, the diagnostic value of plasma miR-181b,
miR-196a, and miR-210 and their combinations in PC has not been fully elucidated.

In this study, the diagnostic roles of plasma miR-181b, miR-196a, and miR-210 in PC were investigated. The 


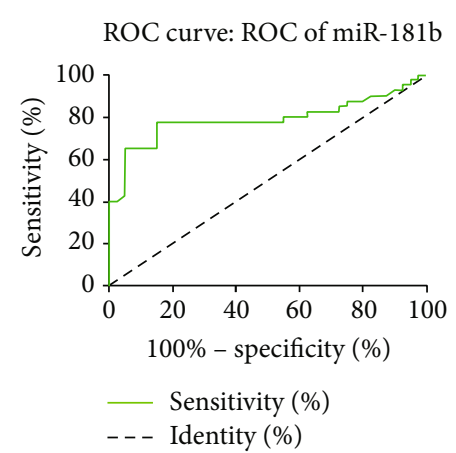

(a)

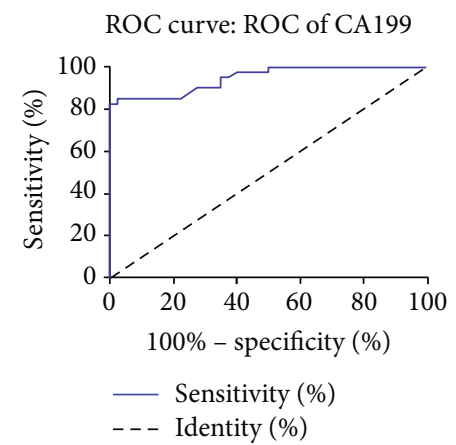

(d)

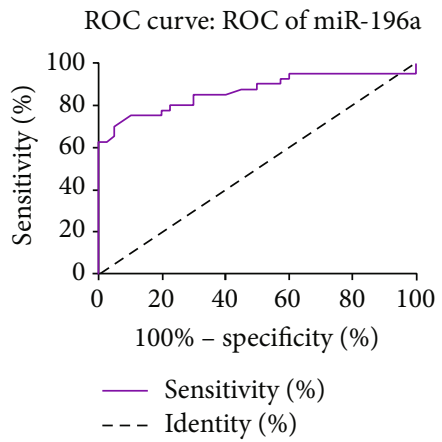

(b)

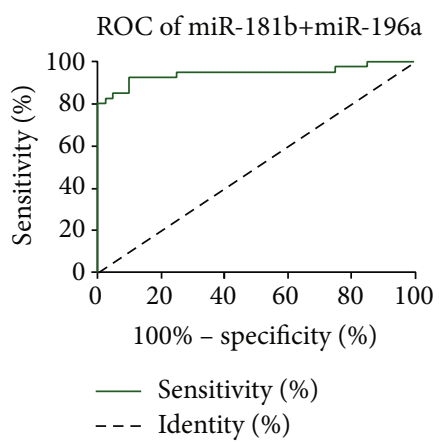

(e)

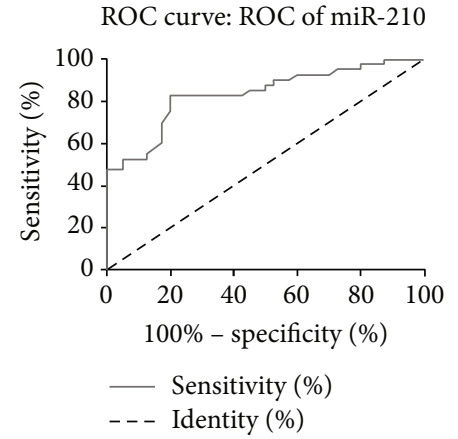

(c)

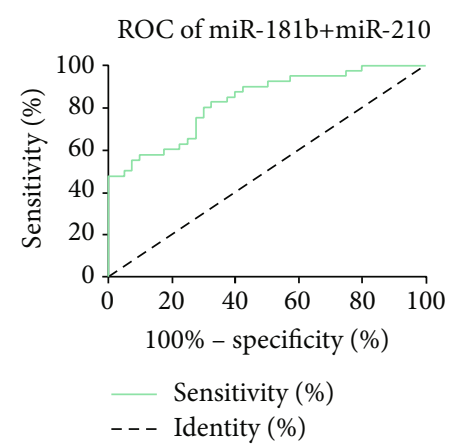

(f)

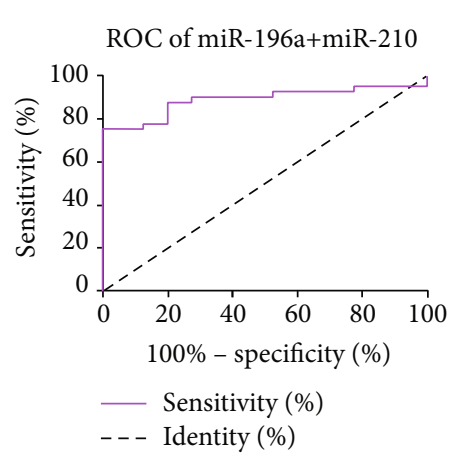

(g)

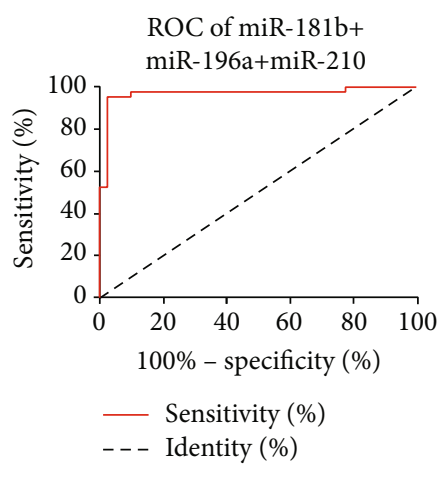

(h)

Figure 2: ROC curves of plasma miR-181b, miR-196a, miR-210, and CA199 in the diagnosis of PC: (a) miR-181b; (b) miR-196a; (c) miR-210; (d) CA199; (e) miR-181b+miR-196a; (f) miR-181b+miR-210; (g) miR-196a+miR-210; (h) miR-181b+miR-196a+miR-210.

expression of plasma miR-181b, miR-196a, and miR-210 was measured by qRT-PCR. The level of the traditional tumor marker carbohydrate antigen 199 (CA199) was detected by an electrochemiluminescence (ECL) assay. In addition, the receiver operating characteristic (ROC) curve was used to determine the diagnostic value of the above plasma miRNAs in PC. We hope to reveal promising biomarkers in the diagnosis of PC.

\section{Methods}

2.1. Clinical Samples. Forty patients with pancreatic ductal adenocarcinoma (22 males and 18 females; $58 \pm 10$ years old; TNM stage: 9 I, 11 II, 13 III, and 6 IV) were recruited in our hospital from May 2016 to January 2019. The inclusion criteria were as follows: (1) first-time diagnosis; (2) no prior history of radiotherapy, chemotherapy, and other adju- vant therapy; and (3) no other malignant tumors. Forty healthy volunteers (22 males and 18 females; $60 \pm 11$ years old) were enrolled as the control. The plasma samples were collected from PC patients and healthy volunteers $(N=40)$. This study was approved by the Ethics Committee of our hospital in accordance with the Declaration of Helsinki. Written informed consent was obtained from patients and volunteers.

2.2. Quantitative Real-Time PCR ( $q R T-P C R)$. Total RNA was extracted from plasma of PC patients and healthy volunteers using TRIzol (Invitrogen, USA). cDNA was then synthesized from total RNA using the Revert Aid First Strand cDNA Synthesis Kit (Thermo, USA). Subsequently, qRT-PCR was performed using the SYBR Green qPCR Master Mix (Thermo Scientific, USA) according to the manufacturer's protocol. U6 was used as the internal control. Primers were 
TABLe 2: The diagnostic value of plasma miR-181b, miR-196a, miR-210, and CA199 in PC.

\begin{tabular}{lccccr}
\hline Markers & Cutoff & $\begin{array}{c}\text { Sensitivity } \\
(\%)\end{array}$ & $\begin{array}{c}\text { Specificity } \\
(\%)\end{array}$ & AUC (area \pm Std.error) & $95 \%$ CI \\
\hline miR-181b & 1.45 & 77.50 & 85.00 & $0.789 \pm 0.055^{* *}$ & $0.681-0.898$ \\
miR-196a & 1.56 & 72.50 & 92.50 & $0.865 \pm 0.044^{*}$ & $0.779-0.951$ \\
miR-210 & 1.46 & 82.50 & 80.00 & $0.834 \pm 0.045^{* *}$ & $0.745-0.923$ \\
CA199 & 41.33 & 82.50 & 99.00 & $0.947 \pm 0.023$ & $<0.0001$ \\
\hline
\end{tabular}

Notes: AUC: area under the curve; CI: confidence intervals. The $P$ value represented the significance of ROC analysis. ${ }^{*} P<0.05,{ }^{* *} P<0.01$ vs. CA199.

TABLE 3: The diagnostic value of plasma miRNA combinations in PC.

\begin{tabular}{|c|c|c|c|c|c|}
\hline Tumor markers & Sensitivity (\%) & Specificity (\%) & AUC (area \pm Std.error) & $95 \% \mathrm{CI}$ & $P$ value \\
\hline miR-181b+miR-196a & 92.50 & 90.00 & $0.944 \pm 0.029$ & $0.887-1.001$ & $<0.0001$ \\
\hline miR-181b+miR-210 & 80.00 & 70.00 & $0.830 \pm 0.045^{* *}$ & $0.743-0.917$ & $<0.0001$ \\
\hline miR-196a+miR-210 & 87.50 & 80.00 & $0.888 \pm 0.042^{*}$ & $0.805-0.970$ & $<0.0001$ \\
\hline $\operatorname{miR}-181 b+196 a+210$ & 95.00 & 97.50 & $0.968 \pm 0.022^{*}$ & $0.924-1.011$ & $<0.0001$ \\
\hline CA199 & 82.50 & 99.00 & $0.947 \pm 0.023$ & $0.902-0.993$ & $<0.0001$ \\
\hline
\end{tabular}

Notes: AUC: area under the curve; CI: confidence intervals. The $P$ value represented the significance of ROC analysis. ${ }^{*} P<0.05,{ }^{* *} P<0.01$ vs. CA199.

as follows: miR-181b: (forward) $5^{\prime}$-GCCGTAAAGTGCTG ACAGT- $3^{\prime}$ and (reverse) $5^{\prime}$-GTGCAGGGTCCGAGGT AT-3'; miR-196a: (forward) 5'-GAAGATCTTTCCTTGG CGGCGACA- $3^{\prime}$ and (reverse) $5^{\prime}$-CCCAAGCTTGATGG CCCGCCTA-3'; miR-210: (forward) 5' -TATACAAGGGC AAGCTCTCTGT-3' and (reverse) $5^{\prime}$-AGAGAGCTTGC CCTTGTATATT-3'; and U6: (forward) $5^{\prime}$-CTCGCTTCG GCAGCACA- $3^{\prime}$ and (reverse) $5^{\prime}$-AACGCTTCACGAAT TTGCGT- $3^{\prime}$.

2.3. ECL Assay. The plasma level of CA199 was measured by the ECL assay using an electrochemical luminescence apparatus (CobasE601, Roche, Sweden). The detection threshold of CA199 was $27 \mathrm{U} / \mathrm{mL}$.

2.4. Statistical Analysis. All statistical analyses were performed using SPSS 22.0 statistical software (Chicago, IL, USA). Data were presented in the form of mean \pm standard deviation (SD). The two-tailed $t$-test was used for comparison between two groups, and one-way ANOVA was used for comparison among multiple groups. The chi-square test was used to compare the qualitative data. The diagnostic value of miRNAs was analyzed by the ROC curve. $P<0.05$ was considered to be statistically significant.

\section{Results}

3.1. The Levels of miR-181b, miR-196a, miR-210, and CA199 Are Increased in PC Patients. Forty PC patients and healthy volunteers were enrolled in this study. There were no significant differences in the age and gender between PC patients and healthy volunteers. The results of qRT-PCR showed that the expression of miR-181b, miR-196a, and miR-210 in the plasma of PC patients was higher than that in healthy volunteers $(P<0.001)$ (Figures $1(\mathrm{a})-1(\mathrm{c}))$. In addition, CA199 level in the plasma of PC patients was significantly higher than that in healthy volunteers $(15.69 \pm 11.37$ vs. $139.40 \pm$ $129.40, P<0.001$ ) (Figure $1(\mathrm{~d})$ ). The above results indicate that the levels of miR-181b, miR-196a, miR-210, and CA199 are increased in PC patients.

3.2. The Expression of $m i R-181 b$, miR-196a, and miR-210 Is Associated with Clinical Parameters in PC. The expression of miR-181b, miR-196a, and miR-210 was significantly correlated with the lymph node metastasis $(P<0.001, P<0.01$, and $P<0.01$, respectively), clinical stage $(P<0.01, P<0.05$, and $P<0.01$, respectively), and vascular invasion $(P<0.001, P<$ 0.01 , and $P<0.01$, respectively) (Table 1 ). However, the expression of miR-181b, miR-196a, and miR-210 had no significant correlation with the age, gender, and tumor size in PC patients $(P>0.05)$ (Table 1$)$.

3.3. Plasma miR-181b, miR-196a, and miR-210 Have a Certain Diagnostic Value in PC. The ROC curve was established to investigate the diagnostic value of plasma markers in PC (Figures 2(a)-2(h)). The results showed that miR181b, miR-196a, and miR-210 have a lower diagnostic value (AUC) than CA199 in PC (Table 2) (all $P<0.05$ ). In addition, the diagnostic value (AUC) of miR-181b+miR-210 and miR-196a+miR-210 in PC was also significantly lower than that of CA199 (Table 3) (all $P<0.05)$. It is worth noting that the combination of plasma miR-181b, miR-196a, and miR-210 (miR-181b+miR-196a+miR-210) has a higher AUC than CA199 in the diagnosis of PC (Table 3) $(P<0.05)$. To sum up, miR-181b+miR-196a+miR-210 (sensitivity: 95.00, specificity: 97.50) improved the diagnostic value of each indicator alone (Table 3 ).

\section{Discussion}

In recent years, the difficulty and delay in the diagnosis of PC are still a therapeutic challenge, which leads to poor prognosis. The main therapeutic methods for PC are surgery, 
chemotherapy, and radiotherapy. Nevertheless, the median survival time is 5 to 8 months, and the 5 -year survival rate $(8 \%)$ is poor [5]. In this study, we explored the diagnostic value of plasma miR-181b, miR-196a, and miR-210 in PC. We found that the combination of plasma miR-181b, miR196a, and miR-210 has a good diagnostic value for PC.

Accumulating evidence has reported that miRNAs play important roles in PC [16-19]. Qiao et al. have shown that miR-381 is a tumor suppressor, which inhibits cell proliferation, migration, and invasion, and induces cell apoptosis in PC [20]. Zhou et al. have reported that miR-340 is downregulated in PC, which can suppress cell growth and reduce tumor size in PC [17]. Xu et al. [21] have indicated that miR-143 is lowly expressed in PC, which can promote cell apoptosis and inhibit cell migration and invasion. In addition, $\mathrm{Xu}$ et al. [21] have also confirmed that miR-143 expression is closely associated with the tumor size, clinical staging, and lymph node metastasis. Previous researches have determined that the expression of miR-196a and miR-210 is upregulated in the plasma of PC patients, indicating that miR-196a and miR-210 may serve as potential biomarkers for PC diagnosis [22]. Ren et al. [15] have reported that fecal miR-181b, miR196a, and miR-210 are highly expressed in PC patients, suggesting that those miRNAs may be biomarkers for PC diagnosis. In the present study, we found that the expression of miR181b, miR-196a, and miR-210 was closely associated with lymph node metastasis, clinical stage, and vascular invasion but not correlated with age, gender, and tumor size in patients with PC. Therefore, we speculate that miR-181b, miR-196a, and miR-210 may be used as biomarkers for PC diagnosis.

Recently, some diagnostic biomarkers in the plasma of PC patients, such as CA199, CA242, MIC-1, and lectins, are accepted for the diagnosis of PC [23-26]. Among these diagnostic biomarkers, CA199 is considered the best available biomarker for PC $[23,27,28]$. Previous studies have shown that the AUC of CA199 in the diagnosis of PC is about 0.80.9 [29-32]. In the present study, the level of CA199 was significantly increased in the plasma of PC patients. The AUC of CA199 in the diagnosis of PC was 0.947, which is higher than that reported previously. This phenomenon may be attributed to the differences in the enrolled subjects. The strict inclusion criteria in this study may enlarge the diagnostic value of CA199. In addition, jaundice-induced increase in CA199 may also disturb the diagnostic value evaluation. Even so, CA199 is not an ideal marker for the diagnosis of $\mathrm{PC}$, especially due to the unsatisfied sensitivity. It is important to explore more effective diagnostic biomarkers for PC. In this study, the expression of miR-181b, miR-196a, and miR-210 was significantly upregulated in PC patients. ROC curves showed that miR-181b, miR-196a, and miR-210 have lower AUC than CA199 in the diagnosis of PC. Studies have confirmed that the combination of plasma miRNAs with CA199 is effective for the diagnosis of PC $[23,33]$. In this study, the combination improved the diagnostic value of each indicator alone in PC. It is worth noting that miR-181b + miR-196a + miR-210 has higher AUC than CA199. These results indicate that the combination of plasma miR-181b, miR-196a, and miR-210 is a promising diagnostic strategy for PC.

\section{Conclusions}

In conclusion, the expression of miR-181b, miR-196a, and miR-210 was significantly upregulated in patients with PC. The combination of plasma miR-181b, miR-196a, and miR210 had a good diagnostic value for PC. Our research provides a theoretical foundation for investigating novel biomarkers for the diagnosis of PC.

\section{Data Availability}

The data used to support the findings of this study are available from the corresponding author upon request.

\section{Conflicts of Interest}

The authors declare that they have no conflict of interest.

\section{Authors' Contributions}

Gongpan Liu had substantial contributions to the conception and design, data acquisition, drafting of the article, and final approval of the version to be published. Cunhua Shao had substantial contributions to the conception and design, data analysis and interpretation, and drafting of the article. Anyun $\mathrm{Li}$ and Xiaobin Zhang drafted the article or were responsible for critically revising it for important intellectual content. Xingjun Guo and Jiangong Li drafted the article or were responsible for critically revising it for important intellectual content. Gongpan Liu and Cunhua Shao were regarded as cofirst authors.

\section{References}

[1] W. Chen, R. Zheng, S. Zhang et al., "The incidences and mortalities of major cancers in China, 2009," Chinese Journal of Cancer, vol. 32, no. 3, pp. 106-112, 2013.

[2] F. X. Real, "A "catastrophic hypothesis" for pancreas cancer progression," Gastroenterology, vol. 124, no. 7, pp. 19581964, 2003.

[3] P. Y. Parikh and K. D. Lillemoe, "Surgical management of pancreatic cancer-distal pancreatectomy," Seminars in Oncology, vol. 42, no. 1, pp. 110-122, 2015.

[4] F. Li, J. W. Xu, L. Wang, H. Liu, Y. Yan, and S. Y. Hu, "MicroRNA-221-3p is up-regulated and serves as a potential biomarker in pancreatic cancer," Artif Cells Nanomed Biotechnol., vol. 46, no. 3, pp. 482-487, 2018.

[5] R. Gupta, I. Amanam, and V. Chung, "Current and future therapies for advanced pancreatic cancer," Journal of Surgical Oncology, vol. 116, no. 1, pp. 25-34, 2017.

[6] R. C. Lee, R. L. Feinbaum, and V. Ambros, "The C. elegans heterochronic gene _lin-4_encodes small RNAs with antisense complementarity to _lin-14_,"Cell, vol. 75, no. 5, pp. 843854, 1993.

[7] B. P. Lewis, C. B. Burge, and D. P. Bartel, "Conserved seed pairing, often flanked by adenosines, indicates that thousands of human genes are microRNA targets," Cell, vol. 120, no. 1, pp. 15-20, 2005.

[8] T. Deng, Y. Yuan, C. Zhang et al., "Identification of circulating miR-25 as a potential biomarker for pancreatic cancer 
diagnosis," Cellular Physiology and Biochemistry, vol. 39, no. 5, pp. 1716-1722, 2016.

[9] M. V. Iorio and C. M. Croce, "MicroRNAs in cancer: small molecules with a huge impact," Journal of Clinical Oncology, vol. 27, no. 34, pp. 5848-5856, 2009.

[10] M. Fabbri, F. Calore, A. Paone, R. Galli, and G. A. Calin, "Epigenetic regulation of miRNAs in cancer," Advances in Experimental Medicine and Biology, vol. 754, pp. 137-148, 2013.

[11] E. J. Duell, L. Lujan-Barroso, N. Sala et al., "Plasma microRNAs as biomarkers of pancreatic cancer risk in a prospective cohort study," Int J Cancer, vol. 141, no. 5, pp. 905-915, 2017.

[12] J. Zhou, J. Wang, X. Chen, W. U. Liming, W. U. Shixing, and W. Wang, "The plasma levels of microRNA-181b in the patients with pancreatic cancer and its clinical significance," Journal of Shanxi Medical University., vol. 40, no. 7, pp. 632633, 2015.

[13] Y.-F. Xu, B. N. Hannafon, Y. D. Zhao, R. G. Postier, and W.Q. Ding, "Plasma exosome miR-196a and miR-1246 are potential indicators of localized pancreatic cancer," Oncotarget, vol. 8, no. 44, pp. 77028-77040, 2017.

[14] A. S. Ho, X. Huang, H. Cao et al., "Circulating miR-210 as a novel hypoxia marker in pancreatic cancer," Translational Oncology, vol. 3, no. 2, pp. 109-113, 2010.

[15] Y. Ren, J. Gao, J. Q. Liu et al., "Differential signature of fecal microRNAs in patients with pancreatic cancer," Molecular Medicine Reports, vol. 6, no. 1, pp. 201-209, 2012.

[16] H. Hu, Q. Zhang, W. Chen et al., "MicroRNA-301a promotes pancreatic cancer invasion and metastasis through the JAK/STAT3 signaling pathway by targeting SOCS5," Carcinogenesis, vol. 41, no. 4, pp. 502-514, 2020.

[17] X. Zhou, Z. Huang, L. Xu et al., "A panel of 13-miRNA signature as a potential biomarker for predicting survival in pancreatic cancer," Oncotarget, vol. 7, no. 43, pp. 69616-69624, 2016.

[18] S. Debernardi, N. J. Massat, T. P. Radon et al., "Noninvasive urinary miRNA biomarkers for early detection of pancreatic adenocarcinoma," American Journal of Cancer Research, vol. 5, no. 11, pp. 3455-3466, 2015.

[19] J. S. Johansen, D. Calatayud, V. Albieri et al., "The potential diagnostic value of serum microRNA signature in patients with pancreatic cancer," International Journal of Cancer, vol. 139, no. 10, pp. 2312-2324, 2016.

[20] G. Qiao, J. Li, J. Wang, Z. Wang, and W. Bian, “miR-381 functions as a tumor suppressor by targeting ETS1 in pancreatic cancer," International Journal of Molecular Medicine, vol. 44, no. 2, pp. 593-607, 2019.

[21] B. Xu, J. Liu, X. Xiang et al., "Expression of miRNA-143 in pancreatic cancer and its clinical significance," Cancer Biotherapy \& Radiopharmaceuticals, vol. 33, no. 9, pp. 373-379, 2018.

[22] Q. Yu, C. Xu, W. Yuan et al., "Evaluation of Plasma MicroRNAs as Diagnostic and Prognostic Biomarkers in Pancreatic Adenocarcinoma: miR-196a and miR-210 Could Be Negative and Positive Prognostic Markers, Respectively," BioMed Research International, vol. 2017, 10 pages, 2017.

[23] J. Liu, J. Gao, Y. Du et al., "Combination of plasma microRNAs with serum CA19-9 for early detection of pancreatic cancer," International Journal of Cancer, vol. 131, no. 3, pp. 683-691, 2012.

[24] Q. Chen, L. Yang, Y. Xiao, J. Zhu, and Z. Li, "Circulating microRNA-182 in plasma and its potential diagnostic and prognostic value for pancreatic cancer," Medical Oncology, vol. 31, no. 11, p. 225, 2014.
[25] T. H. Patwa, J. Zhao, M. A. Anderson, D. M. Simeone, and D. M. Lubman, "Screening of glycosylation patterns in serum using natural glycoprotein microarrays and multi-lectin fluorescence detection," Analytical Chemistry, vol. 78, no. 18, pp. 6411-6421, 2006.

[26] K. Satake, G. Kanazawa, I. Kho, Y. S. Chung, and K. Umeyama, "A clinical evaluation of carbohydrate antigen 19-9 and carcinoembryonic antigen in patients with pancreatic carcinoma," Journal of Surgical Oncology, vol. 29, no. 1, pp. 15-21, 1985.

[27] X. G. Ni, X. F. Bai, Y. L. Mao et al., "The clinical value of serum CEA, CA19-9, and CA242 in the diagnosis and prognosis of pancreatic cancer," European Journal of Surgical Oncology, vol. 31, no. 2, pp. 164-169, 2005.

[28] D. V. Gold, D. E. Modrak, Z. Ying, T. M. Cardillo, R. M. Sharkey, and D. M. Goldenberg, "New MUC1 serum immunoassay differentiates pancreatic cancer from pancreatitis," Journal of Clinical Oncology, vol. 24, no. 2, pp. 252-258, 2006.

[29] Y. Zhang, J. Yang, H. Li, Y. Wu, H. Zhang, and W. Chen, "Tumor markers CA19-9, CA242 and CEA in the diagnosis of pancreatic cancer: a meta-analysis," International Journal of Clinical and Experimental Medicine, vol. 8, no. 7, pp. 11683-11691, 2015.

[30] T. Yoneyama, S. Ohtsuki, K. Honda et al., "Identification of IGFBP2 and IGFBP3 as compensatory biomarkers for CA199 in early-stage pancreatic cancer using a combination of antibody-based and LC-MS/MS-based proteomics," PLoS One, vol. 11, no. 8, article e0161009, 2016.

[31] Y. F. Ma, L. M. He, Q. Wu, and X. Q. Wang, "Detection of chitinase 3-like 1 combined with other biomarkers for diagnosis of pancreatic cancer," Journal of Southern Medical University, vol. 38, no. 4, pp. 450-454, 2018.

[32] F. Qiu, Y. Zhang, and L. Kong, "Combined detection of CA199, CA242 and CA50 in the diagnosis for pancreatic cancer: a meta analysis," Advances in Clinical Medicine., vol. 8, no. 4, pp. 433-445, 2018.

[33] W. S. Wang, L. X. Liu, G. P. Li et al., "Combined serum CA199 and miR-27a-3p in peripheral blood mononuclear cells to diagnose pancreatic cancer," Cancer Prevention Research (Philadelphia, Pa.), vol. 6, no. 4, pp. 331-338, 2013. 